\title{
Electrochemical biosensing of chilled seafood freshness by xanthine oxidase immobilized on copper-based metal-organic framework nanofiber film
}

\begin{abstract}
ATP degradation is an important biochemical change during decomposition of seafood. Hypoxanthine and xanthine which are formed during ATP degradation process can be used for evaluation of chilled seafood freshness. Present study successfully immobilized xanthine oxidase (XOD) onto a type of biocompatible copper-based metal organic framework nanofibers $(\mathrm{Cu}-\mathrm{MOF})$ film and used it for fabrication of a hypoxanthine and xanthine electrochemical biosensor. $\mathrm{Cu}-\mathrm{MOF}$ can efficiently entrap XOD, provide a suitable atmosphere for XOD biocatalysis, and ensure good electron transfer between enzyme and electrode surface. The asprepared XOD-electrochemical biosensor demonstrated high sensitivity for both hypoxanthine and xanthine with a wide linear range $(0.01$ to $10 \mu \mathrm{M})$ and low limit of detection. During the 3-week storage stability test at $4{ }^{\circ} \mathrm{C}$, the fabricated biosensor demonstrated good reusability (up to 100 times) and excellent storage stability for hypoxanthine and xanthine. When applied for detection of hypoxanthine and xanthine in chilled squid and large yellow croaker, the XODelectrochemical biosensors demonstrated good recovery rates.
\end{abstract}

Keyword: Xanthine oxidase; Metal organic framework; Electrochemical properties; Chilled seafood freshness; Biosensor 